\title{
Complement MASP-1 enhances adhesion between endothelial cells and neutrophils by up-regulating E-selectin expression
}

Péter K. Jani ${ }^{\text {a,1, }}$, Endre Schwaner ${ }^{\text {a,1, }}$, Erika Kajdácsi ${ }^{\text {a }}$, Márta L. Debreczeni ${ }^{a}$, Rita Ungai-Salánki ${ }^{\text {b,c,d }}$, József Dobó $^{\mathrm{e}}$, Zoltán Doleschall ${ }^{\mathrm{f}}$, János Rigó Jr. ${ }^{\mathrm{g}}$, Miklós Geiszt ${ }^{\mathrm{h}}$, Bálint Szabóo ${ }^{\mathrm{c}, d}$, Péter Gál ${ }^{\mathrm{e}}$ and László Cervenak $^{\mathrm{a}, *}$

ard Department of Internal Medicine, Semmelweis University, 1125 Budapest, Hungary

${ }^{\mathrm{b}}$ Doctoral School of Molecular- and Nanotechnologies, University of Pannonia, 8200 Veszprém, Hungary;

${ }^{c}$ Nanobiosensorics Lendület Group, Institute for Technical Physics and Materials Science, Centre for Energy Research, 1121 Budapest, Hungary

${ }^{\mathrm{d}}$ Department of Biological Physics, Eötvös Loránd University, 1053 Budapest, Hungary

${ }^{\mathrm{e}}$ Institute of Enzymology, Research Centre for Natural Sciences, Hungarian Academy of Sciences, 1117

Budapest, Hungary

${ }^{\mathrm{f}}$ Department of Pathogenetics, National Institute of Oncology, 1122 Budapest, Hungary

${ }^{\mathrm{g}}$ First Department of Obstetrics and Gynecology, Semmelweis University, 1088 Budapest, Hungary

${ }^{\mathrm{h}}$ Department of Physiology, Semmelweis University, 1094 Budapest, Hungary

Abbreviation: HUVEC, human umbilical vein endothelial cell; MBL, mannan-binding lectin; MASP, mannan-binding lectin-associated serine protease; PAR, protease-activated receptor.

* Corresponding author: Dr. László Cervenak, 3rd Department of Internal Medicine, Semmelweis University, Budapest, Hungary, 1125 Budapest, Kútvölgyi street 4., Hungary. Phone: +36 1 325-1494, Fax: +36 $1212-$ 9351, E-mail address: cervenak.laszlo@med.semmelweis-univ.hu

${ }^{1}$ These authors contributed equally to this work. 


\begin{abstract}
The complement system and neutrophil granulocytes are indispensable in the immune response against extracellular pathogens such as bacteria and fungi. Endothelial cells also participate in antimicrobial immunity largely by regulating the homing of leukocytes through their cytokine production and their pattern of cell surface adhesion molecules. We have previously shown that mannan-binding lectin-associated serine protease-1 (MASP-1), a complement lectin pathway enzyme, is able to activate endothelial cells by cleaving protease activated receptors, which leads to cytokine production and enables neutrophil chemotaxis. Therefore, we aimed to investigate how recombinant MASP-1 (rMASP-1) can modify the pattern of P-selectin, E-selectin, ICAM-1, ICAM-2, and VCAM-1 adhesion molecules in human umbilical vein endothelial cells (HUVEC), and whether these changes can enhance the adherence between endothelial cells and neutrophil granulocyte model cells (differentiated PLB-985). We found that HUVECs activated by rMASP-1 decreased the expression of ICAM-2 and increased that of E-selectin, whereas ICAM-1, VCAM-1 and P-selectin expression remained unchanged. Furthermore, these changes resulted in increased adherence between differentiated PLB-985 cells and endothelial cells. Our finding suggests that complement MASP-1 can increase adhesion between neutrophils and endothelial cells in a direct fashion. This is in agreement with our previous finding that MASP-1 increases the production of pro-inflammatory cytokines (such as IL-6 and IL-8) and chemotaxis, and may thereby boost neutrophil functions. This newly described cooperation between complement lectin pathway and neutrophils via endothelial cells may be an effective tool to enhance the antimicrobial immune response.
\end{abstract}

Keywords: Adhesion, Complement, Endothelial cell, Neutrophil, Inflammation, MASP-1 


\section{Introduction}

The effectiveness of the immune system depends on the prompt and specific response against pathogens. Therefore, it is not surprising that immunological subsystems cooperate with one another for the quick recognition and elimination of invaders or altered host cells. A good example of this cooperation is the teamwork of the complement system and neutrophil granulocytes against bacteria and fungi.

The complement system can be activated through different routes: the classical, the alternative, and the lectin pathways. After recognition of the target structure by any of the three pathways, a cascade of serine proteases is activated, and this leads to the cleavage of $\mathrm{C} 3$, the central molecule of the complement system. $\mathrm{C} 3 \mathrm{~b}, \mathrm{C} 4 \mathrm{~b}$ and their degradation fragments act as opsonins enhancing the phagocytic activity of neutrophils (and other cell types). C3a and C5a are amongst the most potent chemotactic factors for neutrophils attracting them to the site of the infection.

Mannan-binding lectin (MBL)-associated serine protease-1 (MASP-1) is the primary enzyme of the lectin pathway [1-3]. MASP-1 is activated upon the recognition of special pathogen/danger-associated motifs by MBL, collectin-11 (CL-K1), or ficolins (1-, 2-, and 3-ficolin) [4, 5]. Following its autoactivation, MASP-1 cleaves MASP-2 and C2, thereby ensuring further activation of the lectin pathway. However, we and other groups have described several other functions of MASP-1 beyond complement activation [6]. MASP-1 can cleave high-molecular-weight kininogen to bradykinin, a vasoactive and nociceptive peptide [7]. It can also cleave prothrombin, FXIII and TAFI, thus MASP-1 can modulate the function of the clotting and fibrinolytic systems [8-10]. Moreover, active MASP-1 (both in native form complexed with MBL and as recombinant, rMASP-1) is able to cleave protease activated receptors (PAR1, 2 and 4), and this generates a proinflammatory signal in endothelial cells $[11,12]$. Upon this stimulus, the endothelial cells produce IL- 6 and IL-8 and thus, recruit neutrophil granulocytes [13].

Endothelial cells are vascular site specific regulators of several physiological processes [14, 15]. To interact with circulating blood cells, endothelial cells must utilize a wide range of adhesion molecules. These adhesion molecules can be classified according to their structure, subcellular localization, and function. Upon traumatic vascular injury, endothelial cells translocate their pre-formed P-selectin molecules onto the cell surface by degranulation occurring within a few minutes [16, 17]. P-selectin is the major lectin-type adhesion molecule of endothelial cells that interacts with platelets to ensure their adherence to the injured vessel wall. Inflammation induced by invading microorganisms or necrotic debris stimulates endothelial cells to modify the pattern of other adhesion molecules. Sialyl-Lewis $\mathrm{X}$ recognizing E-selectin is de novo synthesized by endothelial cells within hours of induction to enhance the initial adhesion and rolling of leukocytes on the endothelium [18, 19]. ICAM-1, a receptor of beta- 2 integrins, is expressed on the surface of most endothelial cells (and of some leukocytes) at a low level; however, its expression is up-regulated within 12-24 hours in response to pro-inflammatory stimuli [20-22]. It participates in the transmigration of all types of leukocytes. VCAM-1 is another inflammatory adhesion molecule synthesized de novo, but only later (24 hours after) an appropriate stimulus. As a partner of VLA-4, it has a major role in the homing of Tcells and monocytes, as well as subsets of neutrophils also use this adhesion molecule [23, 24]. ICAM-2 is an endothelial cell specific adhesion molecule. Although it is constitutively expressed on non-activated endothelial cells, its exact role is still not fully understood. ICAM-1 and ICAM-2 share their integrin ligands. However, the affinity spectrum of ICAMs towards different beta-2 integrins differ, and ICAM-2 does not induce outside-in signaling of integrin partner molecules [25, 26]. ICAM-2 expression decreases upon proinflammatory stimuli [27].

To control leukocyte homing during inflammation, the production of cytokines and the expression of adhesion molecules are usually co-regulated in endothelial cells. We have previously described that rMASP-1 can induce IL-6 and IL-8 production in HUVECs, which is regulated predominantly by the p38- 
MAPK pathway [13]. Therefore, in this study we investigated how rMASP-1 can modify the pattern of P-selectin, E-selectin, ICAM-1, ICAM-2, and VCAM-1 adhesion molecules in endothelial cells. We also explored whether these changes can enhance the adherence between endothelial cells and neutrophil granulocytes.

\section{Material and methods}

\subsection{Reagents}

We used recombinant catalytic fragment of human MASP-1 (CCP1-CCP2-SP, hereinafter: rMASP-1). rMASP-1 was expressed in E. coli as described by Ambrus et al. [28], and prepared according to Dobó et al. [29]. Briefly, after dissolving inclusion bodies and refolding in glutathion/oxidized glutathion containing buffer for one month, rMASP-1 autoactivated during dialysis. Then sequential ion-exchange chromatographies of rMASP-1 were performed using anion-exchange (Source 30Q, GE Healthcare) and cation-exchange (SP Sepharose High Performance, GE Healthcare) columns. Purity was checked by PAGE, whereas enzymatic activity was measured by SLGR-AMC substrate cleaving assay. The rMASP-1 preparations were free of bacterial contaminations, since its NFKB nuclear translocating activity could not be blocked with Polymyxin B or DNase, unlike control LPS and bacterial DNA. Furthermore, it could be inhibited by C1-Inhibitor as described previously [11-13]. The mouse anti-human P- and E-selectin, ICAM-1, ICAM-2 and VCAM-1 antibodies were purchased from Bender MedSystems (Affimetrix, Inc., San Diego). The FITC-conjugated mouse anti-human LFA-1 (CD11a/CD18), MAC-1 (CD11b/CD18), CD15 and CD49d antibodies were purchased from ImmunoTools (ImmunoTools GmbH, Germany). The Alexa Fluor ${ }^{868}$-conjugated goat anti-mouse secondary antibody was purchased from Invitrogen (Invitrogen Co., Oregon, USA) and the horseradish-peroxidase (HRP) conjugated secondary antibody was obtained from Southern Biotech (SouthernBiotech, Birmingham, USA). The recombinant human E-selectin was supplied by Sino Biological (Sino Bilogical Inc., Bejing, P.R. China). All other reagents were purchased from Sigma-Aldrich, unless otherwise stated.

\subsection{Preparation and culturing of human umbilical vein endothelial cells (HUVECs)}

Cells were harvested from fresh umbilical cords obtained during normal deliveries of healthy neonates by collagenase digestion as described earlier [13, 30]. HUVECs were kept in gelatin-precoated flasks (Corning® Costar®) in MCDB131 medium (Life Technologies) completed with 5\% heat-inactivated fetal calf serum (FCS), $2 \mathrm{ng} / \mathrm{ml}$ human recombinant epidermal growth factor (R\&D Systems), $1 \mathrm{ng} / \mathrm{ml}$ human recombinant basic fibroblast growth factor (Sigma), $0.3 \%$ Insulin Transferrin Selenium (Life Technologies), $1 \%$ Chemically Defined Lipid Concentrate (Life Technologies), 1\% Glutamax (Life Technologies), 1\% Penicillin-Streptomycin antibiotics (Sigma), $5 \mu \mathrm{g} / \mathrm{ml}$ Ascorbic acid (Sigma), $250 \mathrm{nM}$ Hydrocortisone (Sigma), $10 \mathrm{mM}$ Hepes (Sigma), and $7.5 \mathrm{U} / \mathrm{ml}$ Heparin (this completed medium is hereinafter: Comp-MCDB). Each experiment was performed on at least three independent primary HUVEC cultures from different individuals before the 4 th passage. The study was conducted in conformity with the WMA Declaration of Helsinki; its protocol was approved by the Semmelweis University Institutional Review Board (permission number: TUKEB64/2008), and all participants provided their written informed consent before inclusion.

\subsection{Culturing of the PLB-985 cell line, as a model for neutrophils}

PLB-985 cells were grown in RPMI-1640 medium (Life Technologies) supplemented with 10\% FCS, $1 \%$ PEST, and 1\% Glutamax. Then, the cells were differentiated into neutrophil-like cells for 6 days in 
the presence of $1.25 \%$ dimethyl sulfoxide (DMSO) [31] (the differentiated PLB-985 cells are hereinafter referred to as dPLB-985 cells). Differentiation was checked by detecting CD11b, CD11a, CD15, CD43 and CD49d expression, and by ascertaining the capability of the cells to generate hydrogen-peroxide.

\subsection{Flow cytometry analysis}

$4 \times 10^{6}$ PLB-985 or dPLB-985 cells were washed and resuspended in HBSS. To detect CD11a, CD11b, CD15, CD43 and CD49d expression, $5 \mu \mathrm{g}$ of appropriate FITC conjugated antibodies, or the isotype control antibodies were added to the cell suspensions ( $5 \times 10^{5}$ cells in $\left.50 \mu \mathrm{PBS}\right)$ and incubated for $30 \mathrm{~min}$ at $4{ }^{\circ} \mathrm{C}$. The samples were then washed in $250 \mu \mathrm{l}$ cold PBS containing $0.1 \%$ BSA, centrifuged and resuspended/fixed in PBS containing $2 \%$ formaldehyde for 15 minutes. Next, the samples were washed in $250 \mu \mathrm{L}$ PBS, centrifuged and resuspended in $300 \mu \mathrm{L}$ PBS. Cell surface expression of adhesion molecules was monitored with a FACScalibur flow cytometer (Becton Dickinson, Franklin Lakes, NJ). No gates were used except FSC threshold avoiding debris and other microparticles. The FACS figures were plotted using Flowing Software 2.5 (www.flowingsoftware.com).

\subsection{Detection of hydrogen peroxide production}

An Amplex Ultra Red (Life Technologies) based fluorescence method was used [32] to quantitate the production of reactive oxygen species. In brief, $2 \times 10^{4}$ cells were activated with $100 \mathrm{nM}$ phorbol 12 myristate 13-acetate (PMA) or left untreated as controls. Then, fluorescence was recorded continuously for 20 minutes, and expressed as arbitrary units.

\section{6. $m R N A$ analysis}

Following pre-treatment by various methods, HUVECs were lysed and stored in TRI@ reagent. Total RNA purification, reverse transcription and LightCycler® analysis were performed as described earlier [13, 30]. The primers (Table 1) were designed with Primer3 (v. 0.4.0) designer from the NCBI database, and produced by Bio Basic Canada Inc.. $\beta$-actin and GAPDH gene-specific primers were used as internal controls. The purity and the size of the PCR products were checked by melting curve analysis, agarose gelelectrophoresis, and sequencing.

\subsection{Visualization of adhesion molecules by immunofluorescence microscopy}

Confluent layers $\left(10^{4}\right.$ cell/well) of HUVECs were cultured in 96-well plates (Corning ${ }^{\circledR}$ Costar $\left.{ }^{\circledR}\right)$ for one day, then the cells were treated with $2 \mu \mathrm{M}$ of rMASP-1, $300 \mathrm{nM}$ thrombin, $50 \mu \mathrm{M}$ histamine or $10 \mathrm{ng} / \mathrm{mL}$ TNFalpha in $100 \mu \mathrm{l} \mathrm{Comp-MCDB}$, or left untreated. After 5 minutes, 6 or 24 hours, the cells were fixed in $1 \%$ formaldehyde for $15 \mathrm{~min}$ (for P-selectin), or in methanol-acetone (1:1) for $10 \mathrm{~min}$ (for other adhesion molecules). Then the cells were stained with primary anti-human antibodies as indicated (all antibodies were diluted 1:500), followed by Alexa Fluor ${ }^{\circledR 568-c o n j u g a t e d ~ g o a t ~ a n t i-m o u s e ~ I g G ~(1: 500), ~ a n d ~ H o e c h s t ~} 33342$ (1:50000, Molecular Probes/Invitrogen). The preparations were read using an Olympus IX-81 fluorescence inverted microscope as described previously $[13,33]$.

\subsection{Measurement of adhesion molecule expression by cell-based ELISA}

Confluent layers of HUVECs were cultured in 96-well plates for 24 hours. Then, HUVECs were treated with $0.2,0.6$, or $2 \mu \mathrm{M}$ rMASP-1, $300 \mathrm{nM}$ thrombin, or $10 \mathrm{ng} / \mathrm{mL}$ TNFalpha for $1,3,6,10$ or 24 hours. The cells were fixed and stained with P-selectin, E-selectin, ICAM-1, ICAM-2, or VCAM-1 antibodies for 60 minutes at room temperature. Then, HRP-conjugated goat anti-mouse antibody and 3,3',5,5'-Tetra Methyl Benzidine (TMB) (Life Technologies) were used to measure the expression of adhesion molecules. 


\subsection{Measurement of PLB-985 and dPLB-985 cell adhesion to E-selectin coated plates}

96-well plates were coated with $0.2,1$, or $5 \mu \mathrm{g} / \mathrm{mL}$ recombinant human E-selectin (Sino Biological Inc.), or left uncoated for 1 hours at $37^{\circ} \mathrm{C}$, and then blocked with PBS containing 2\% BSA for 1 hour at $37^{\circ} \mathrm{C}$, in $5 \% \mathrm{CO}_{2}$ atmosphere. PLB-985 or dPLB-985 cells were incubated in HBSS containing $2 \mu \mathrm{g} / \mathrm{mL}$ Oregon Green ${ }^{\circledR} 488$ carboxylic acid diacetate succinimidyl ester (abbreviated as OG-488, Invitrogen) for 30 min in the dark, at $37{ }^{\circ} \mathrm{C}$, in $5 \% \mathrm{CO}_{2}$ atmosphere. Then, the cells were centrifuged, and resuspended in HBSS for 30 min. OG-488 labeled PLB-985 or dPLB-985 cell suspension was added to the E-selectin precoated wells (10000 cells/well), and incubated for 1 hour, at $37^{\circ} \mathrm{C}$, in $5 \% \mathrm{CO}_{2}$ atmosphere. Then, total number of dPLB-985 cells was determined with a fluorescence plate reader (Tecan Infinite ${ }^{\circledR}$ M1000 Pro), at $485 \mathrm{~nm}$ excitation and $525 \mathrm{~nm}$ emission wavelengths. Thereafter, the plate was washed twice vigorously with HBSS, and the fluorescence of bound cells was monitored again. The number of bound cells normalized with the initial number of cells was calculated after three independent measurements.

\subsection{Assessing dPLB-985 cell adhesion to HUVECs}

Confluent layers of HUVECs were cultured in 96-well plates and treated for 6 or 24 hours with 0.2, 0.6, or $2 \mu \mathrm{M}$ rMASP-1, $300 \mathrm{nM}$ thrombin, or $10 \mathrm{ng} / \mathrm{mL}$ TNFalpha. Then, $2 \times 10^{4}$ OG-488 labeled dPLB-985 cells/well were applied to the HUVECs, and incubated for 1 hour at $37^{\circ} \mathrm{C}$, in $5 \% \mathrm{CO}_{2}$ atmosphere. Total dPLB-985 cell number was monitored by fluorescence as described before. Thereafter, the plate was washed twice vigorously with HBSS using a multichannel pipettor, and the fluorescence of bound cells was monitored again. The ratio of bound to total cell numbers was calculated after three independent measurements. In some experiments, HUVECs were pre-incubated for 30 minutes with the following pathway inhibitors: $25 \mu \mathrm{M}$ JNK inhibitor (SP600125), $2 \mu \mathrm{M}$ p38-MAPK inhibitor (SB203580), $1 \mu \mathrm{M}$ ERK $1 / 2$ inhibitor (U0126) or, $5 \mu \mathrm{M}$ NfkappaB inhibitor (Bay-11 7082). Furthermore in some cases OG-488labeled dPLB-985 cells were pre-treated with $1 \mu \mathrm{g} / \mathrm{mL}$ recombinant E-selectin for 30 minutes, at $37^{\circ} \mathrm{C}$, in $5 \% \mathrm{CO}_{2}$ atmosphere, before adding to the HUVECs, in order to assess the inhibition of adhesion.

\subsection{Measurement of the adhesion force between dPLB-985 cells and endothelial cells with a computer controlled micropipette method}

A computer controlled micropipette (CellSorter), mounted onto an inverted fluorescence microscope (Zeiss Axio Observer A1) [34, 35], was used to quantify the adhesion force between the variously pre-treated HUVECs and dPLB-985 cells. In brief, a confluent layer of HUVECs was cultured in a 35-mm tissue culture plastic Petri dish (Greiner) for one day. Then, they were treated/not treated with $2 \mu \mathrm{M}$ of rMASP-1, $300 \mathrm{nM}$ thrombin for 6 hours, or with $10 \mathrm{ng} / \mathrm{mL}$ TNFalpha for 24 hours. $10^{5}$ OG-488 labeled dPLB-985 cells were placed into the Petri dish containing the endothelial cells, and incubated for 60 minutes at room temperature. Attached dPLB-985 cells were scanned and recognized using the CellSorter software. The adhesion force was measured with a glass micropipette with an inner diameter of $70 \mu \mathrm{m}$. The micropipette was automatically positioned to 100 selected cells (diameter $\sim 13 \mu \mathrm{m}$ ) one by one, in order to try to pick them up (Fig. 5A). Repeating this cycle (i.e. the positioning and pick-up process) each time on the same path with an increased vacuum (expressed in $\mathrm{kPa}$ ), the adhesion force of cells could be precisely measured. The number of the cells was counted before and after each cycle, and the ratio of adherent to originally selected cells was calculated.

\subsection{Statistical analysis}

Statistical analyses were performed using Student's $t$-test, one-way ANOVA with Tukey's post-test or a post-test for linear trends, or two-way ANOVA with Bonferroni post-test (GraphPad Prism 5.01 
software, GraphPad, http://www.graphpad.com/). A p-value less than 0.05 was considered statistically significant. The data are presented as means \pm SEM unless otherwise stated.

\section{Results}

\subsection{Screening the mRNA expression of adhesion molecules}

Since pro-inflammatory adhesion molecules are usually synthesized de novo upon stimuli, we measured the mRNA levels of adhesion molecules by qPCR - except in the case of P-selectin, which is stored as a preformed protein in Weibel-Palade bodies.

Confluent layers of HUVECs were treated with $2 \mu \mathrm{M}$ rMASP-1 for 1, 2, 6 or 10 hours, to assess mRNA expression. rMASP-1 greatly up-regulated the mRNA expression of E-selectin and VCAM-1 in endothelial cells, although the increment was somewhat less in the latter. Maximum effect was observed between 2 and 6 hours and then, the expression of both adhesion molecules declined. The level of ICAM-2 mRNA decreased until the end of the measurement, whereas the expression of ICAM-1 mRNA did not change upon rMASP-1 stimulation (Fig. 1A).

\subsection{The effect of rMASP-1 on the expression of cell adhesion molecules}

The altered expression of these adhesion molecules indicates the pro-inflammatory changes occurring in endothelial cells. Moreover, the effects of known endothelial cell activators (such as thrombin, TNFalpha or histamine) are well characterized with regard to adhesion molecules.

Thrombin and histamine induced prompt P-selectin expression at 5 minutes, and reuptake by endothelial cells within 60 minutes; however, rMASP-1 failed to increase P-selectin expression (Fig. 1, B and C).

Fluorescence microscopy showed that rMASP-1, thrombin, and TNFalpha induced the expression of E-selectin at 6 hours (Fig. 1B). Using cell-based ELISA, we demonstrated that the effect of rMASP-1 on Eselectin expression declined by 24 hours, similar to that of thrombin, but unlike to that of TNFalpha (Fig. 1C). rMASP-1 did not modify the expression of ICAM-1 at any time (Fig. 1, B and C). By contrast, both thrombin and TNFalpha elevated the level of ICAM-1, although with different kinetics (Fig. 1C). rMASP-1, thrombin, and TNFalpha all reduced ICAM-2 expression at 6 hours, and even further at 24 hours (Fig. 1, B and C). Interestingly, although rMASP-1 significantly induced the expression of VCAM-1 mRNA, it had no effect on VCAM-1 protein level (Fig. 1, B and C). Thrombin behaved similar to rMASP-1 in respect of VCAM-1 protein expression; however, TNFalpha induced VCAM-1 at 6 hours, and this effect was even more pronounced at 24 hours (Fig. 1, B and C).

\section{3. rMASP-1 dose dependence and the kinetics of E-selectin and ICAM-2 expression}

It is known that the expression kinetics of various adhesion molecules depends on the inherent properties of the adhesion molecule itself, and on those of the activator. The difference in expression kinetics can regulate selective cell adhesion. To assess the influence of rMASP-1 kinetics on the expression of adhesion molecules at protein level, HUVECs were treated with $2 \mu \mathrm{M}$ rMASP-1 for 1, 3, 6, 10 and 24 hours and then, cell-based ELISA was performed. Similar to the kinetics seen at mRNA level (Fig. 1A), the protein expression of E-selectin was increased as early as at 3 hours, but the maximum effect was observed at 6 hours. E-selectin expression declined until 24 hours post treatment (Fig. 2A). In contrast to E-selectin, ICAM-2 level was lower at 3 hours than that of the untreated control, and decreased continuously until 24 hours (Fig. 2B). Then, at the times of maximum rMASP-1 effect, we assessed dose dependence. We found that treatment with rMASP-1 induced E-selectin and reduced ICAM-2 expression in a dose-dependent manner (Fig. 2, C and D). 


\subsection{Measurement of adhesion between dPLB-985 cells and endothelial cells}

To clarify the biological importance of the previously described alterations of endothelial adhesion molecules, we assessed whether these molecules can bind to neutrophil granulocytes. In these adhesion measurements, we used the acute myeloid leukemia cell line PLB-985, differentiated with DMSO toward neutrophil-like cells (dPLB-985). To verify the effectiveness of the differentiation of dPLB-985 cells, we tested the cell surface expression of adhesion molecules, and the generation of reactive oxygen species. dPLB-985 cells expressed CD11a and CD11b in increased quantities, and reduced amount of CD49d compared to non-differentiated PLB-985 cells. The expression of CD15 and CD43 did not change significantly (Fig. 3A). Moreover, dPLB-985 cells, unlike their ancestors, generated reactive oxygen species in response to $100 \mathrm{nM}$ PMA, as measured by $\mathrm{H}_{2} \mathrm{O}_{2}$ production (Fig. 3B).

To test the adhesive capacity of dPLB-985 cells to E-selectin, microwell plates were coated with 0.2 , 1 , or $5 \mu \mathrm{g} / \mathrm{mL}$ recombinant E-selectin, or left untreated. Then, OG-488-labeled PLB-985 or dPLB-985 cells were added, incubated for 1 hour, washed, and the number of adherent cells was calculated. The adhesion of dPLB-985 cells to the coated plate was dose-dependent, and treatment with E-selectin in a concentration as low as $5 \mu \mathrm{g} / \mathrm{mL}$ resulted in significant adhesion compared to the uncoated controls. We did not observe similar dose-dependent adhesion of non-differentiated PLB cells to E-selectin coated plates (Fig. 4A).

Since we demonstrated that rMASP-1 treatment induces E-selectin expression in HUVECs, and dPLB-985 cells can adhere to recombinant E-selectin, we assessed the adherence between dPLB-985 cells and HUVECs treated with rMASP-1. To this end, HUVECs were treated with $0.2,0.6$, or $2 \mu \mathrm{M}$ rMASP-1, $300 \mathrm{nM}$ thrombin or $10 \mathrm{ng} / \mathrm{mL}$ TNFalpha for 6 or 24 hours, or left untreated. Then OG-488 labeled dPLB-985 cells were applied onto the HUVECs and incubated for 1 hour. The number of adhered dPLB-985 cells was measured by fluorescence, and normalized with baseline total cell number. We observed that rMASP-1 treatment increased dPLB-985 cell adhesion to HUVECs in a dose dependent manner at 6 hours, but not at 24 hours (Fig. 4B). Both the kinetics and the volume of this effect were similar for rMASP-1 and for thrombin. In contrast, TNFalpha induced enhanced adhesion to HUVECs at 6 hours, but adherence was even greater by 24 hours (Fig. 4C). The adhesion between dPLB-985 cells and rMASP-1-induced HUVECs could be reduced to the level of untreated HUVECs by pre-incubating the dPLB-985 cells with $1 \mu \mathrm{g} / \mathrm{mL}$ soluble recombinant E-selectin for 30 minutes (Fig. 4D).

Expression of adhesion molecules can be triggered through various signaling pathways. We reported that most of these pathways can also be activated by rMASP-1 in HUVECs [13]. We used commercially available signaling pathway inhibitors to identify the most important pathways required for the E-selectin mediated adhesion between dPLB-985 cells and rMASP-1-induced HUVECs. HUVECs had been preincubated with the inhibitors for 30 minutes and then, treated with $2 \mu \mathrm{M}$ rMASP-1 for 6 hours. Only the p38MAPK inhibitor was able to block this adhesion, whereas JNK, NFkappaB, and ERK 1/2 pathway inhibitors had no blocking effect (Fig. 4D).

We used a computer controlled micropipette method to quantify the adhesion force between dPLB985 cells and rMASP-1-treated HUVECs [34, 35]. HUVECs were induced with $2 \mu \mathrm{M}$ of rMASP-1, $300 \mathrm{nM}$ thrombin for 6 hours, or $10 \mathrm{ng} / \mathrm{mL}$ TNFalpha for 24 hours or left untreated. Then, OG-488-labeled dPLB-985 cells were added and incubated for 1 hour. The attached dPLB-985 cells were probed (i.e., by trying to pick them up) with the automated micropipette. The hydrodynamic lifting force, acting on the targeted single cells, was increased in each subsequent cycle of the measurement (Fig. 5A). More dPLB-985 cells stayed attached to rMASP-1-treated endothelial cells than to non-treated controls at higher detaching forces. We observed the same effect after thrombin treatment (Fig. 5B). Induction with TNFalpha for 24 hours, used as positive control, was the most potent in enhancing adhesion (by 4.28 -fold, $\pm 1.63, \mathrm{p}<0.01$ at $19.2 \mathrm{kPa}$ vacuum). 


\section{Discussion}

Infection and tissue damage initiate pro-inflammatory activation of the endothelium. This modification of the endothelial cell phenotype includes altered cytokine production and adhesion molecule pattern. In the present study, we found that rMASP-1 treatment changed the adhesion molecule pattern, reduced the expression of ICAM-2, and increased that of E-selectin, whereas ICAM-1, VCAM-1 and Pselectin did not change. Furthermore, these alterations increased the adherence between neutrophil model cells (dPLB-985) and endothelial cells.

The migration of different leukocyte subsets depends, in the first place, on the pattern of chemokines and adhesion molecules produced by the endothelium. Earlier, we have demonstrated that rMASP-1 induces pro-inflammatory processes in endothelial cells. In particular, they activate $\mathrm{Ca}^{2+}, \mathrm{NF} \kappa \mathrm{B}, \mathrm{p} 38-\mathrm{MAPK}, \mathrm{JNK}$, and CREB signaling pathways by cleaving PARs [11, 13]. Moreover, rMASP-1-stimulated endothelial cells produce IL-6 and IL-8, as well as recruit neutrophil granulocytes [13]. Therefore, our previous and current results together imply a novel, endothelial cell mediated pro-inflammatory role for rMASP-1.

Although MASP-1 is the most abundant protease of the lectin pathway, its isolation from human serum is a low-yield process, and the produced protein is prone to degradation during the purification. The catalytic region of MASP-1, containing the CCP1, CCP2 and SP domains, is enzymatically equivalent to the fulllength molecule. Structural studies showed that only the catalytic region is accessible to the substrates present in MBL-MASP-1 complexes, since only the CCP1-CCP2-SP domains protrude from the plane of MBL [36]. Previously, we demonstrated equivalent cellular effects of the serum purified MBL-MASP complex, and of the recombinant catalytic fragment of human MASP-1. Serum purified MASP-1 and rMASP-1 used in equimolar concentration induced the same intensity of Ca-mobilization, and both could be completely blocked with C1-inhibitor [12]. In the current study, we observed significant effects of MASP-1 on adhesion molecules and adhesion between 0.2 and $2 \mu \mathrm{M}$, which is higher than the plasma concentration of MASP-1 ( 143 nM). However, at the site of injury and/or infection, MASP-1 may be trapped via the pattern recognition of MBL and ficolins, thus the local concentration may be much higher than its plasma concentration.

P-selectin, one of the best-characterized components of Weibel-Palade bodies [16], is released rapidly in response to among others thrombin and histamine by G-protein coupled PARs [37], and by histamine H1 receptors [38], respectively. Although it plays a role in the inflammatory response by mediating the rolling of leukocytes on the endothelium [38], the most critical function of P-selectin is to facilitate platelet aggregation at the sites of vascular injury [39]. In contrast to P-selectin, E-selectin is an endothelial cellspecific adhesion molecule; it recognizes sugar motifs containing sialyl-Lewis $\mathrm{X}$ on leukocytes, and has an important role in the initial step of the endothelium/leukocyte adhesion - leukocyte rolling [40, 41]. Eselectin is not detectable on the surface of normally functioning endothelial cells and it is also absent from their cytoplasm; however, upon pro-inflammatory stimuli (e.g. by TNFalpha, LPS, IL-1 $\beta$ ), it is synthesized de novo within hours [42]. Our results indicate that rMASP-1 alone possibly cannot influence the endothelial cell/platelet interaction, but it may regulate the rolling of leukocytes on endothelial cells via the upregulation of E-selectin.

ICAM-1 and ICAM-2 are members of the Ig superfamily; they bind $\beta_{2}$-integrins $(\mathrm{CD} 11 \mathrm{a} / \mathrm{CD} 18$, $\mathrm{CD} 11 \mathrm{~b} / \mathrm{CD} 18$ and CD11c/CD18) with different affinity [25]. ICAM-1 is expressed at a low level on endothelial cells with a non-inflammatory phenotype, as well as on different leukocyte subsets, and their expression is induced - similar to E-selectin - by pro-inflammatory factors [33]. Unlike ICAM-1, ICAM-2 is specific to endothelial cells, as well as it is constitutively and strongly expressed on normally functioning 
cells. Upon pro-inflammatory stimulation, the cells reduce its expression in parallel with the elevation of ICAM-1 levels. Further, their outside-in signaling is different, as the crosslinking of ICAM-1 induces actin reorganization via RhoA. Thus, it participates in the cellular movements of endothelial cells during leukocyte transmigration, whereas ICAM-2 lacks this function [26]. VCAM-1, another endothelial cell-specific adhesion molecule expressed upon pro-inflammatory stimuli, reaches its maximum 24 hours after activation; it is a ligand for VLA-4 and other $\beta_{1}$-integrins [23]. VCAM-1 is required for the transmigration of T cells and monocytes; however, neutrophils may also use the VLA-4/VCAM-1 adhesion for homing [43]. rMASP1 did not induce the expression of ICAM-1 nor VCAM-1 and thus, it may not have a direct influence on the firm contact and on the transmigration of the leukocytes through the endothelium. However, by decreasing ICAM-2, it biases the ICAM-1 to ICAM-2 ratio, and this may potentiate transmigration upon other synergistic pro-inflammatory stimuli (such as by LPS, TNFalpha or C5a). Moreover, the elevation of VCAM-1 expression only at mRNA level also suggests a potential for synergism. The discrepancy between mRNA and protein levels of VCAM-1 is not unknown [44, 45]. MicroRNAs may block translation of VCAM-1 without degradation of mRNA or post-translational degradation can decrease the protein level, however, the exploration of the molecular mechanism is beyond the scope of this study.

rMASP-1 induced a unique pattern and kinetics of adhesion molecules that differs from those induced by TNFalpha and thrombin. The dissimilarity between rMASP-1 and TNFalpha is not surprising, because TNFR strongly activates NFkB, p38-MAPK and JNK signaling pathways [33]. Further, it does not trigger Ca-mobilization, whereas PARs cleaved by rMASP-1 induce Ca-mobilization, JNK and p38-MAPK phosphorylation, but only weak NFאB nuclear translocation [11, 13]. More interestingly, the rMASP-1- and thrombin-induced patterns of adhesion molecules were also different. The expression of P-selectin and of ICAM-1 was enhanced by thrombin, but not by rMASP-1 - this can be explained by the different utilization of PARs. While thrombin cleaves PAR-1 and - to a lesser extent - PAR-4, rMASP-1 is most effective in cleaving PAR-4, followed by PAR-2 and PAR-1 [11]. If we consider the expression profile of different PARs on endothelial cells (PAR-2 expression is the highest, followed by PAR-1, and - to a much less extent - by PAR-4) [11], only partial overlap can be supposed between thrombin and rMASP-1 signaling. Differential signal transduction by the various PARs is well known and in support of our findings [46]. Since we have previously found that in the case of rMASP-1, the cytokine production was regulated predominantly by p38-MAPK pathway [13], it is not unexpected that only the p38-MAPK inhibitor was able to block adhesion between dPLB-985 cells and rMASP-1-induced HUVECs.

Since rMASP-1-treated HUVECs produce IL-8 but not MCP-1 [13], and as we have found an elevated level of E-selectin, but not of ICAM-1 or of VCAM-1, we propose that rMASP-1 induced an endothelial cell phenotype most suitable for the adhesion of neutrophils. Nevertheless, we could not exclude the possibility that other adhesion molecules than E-selectin, which were not measured in this study, are also involved in the increased adhesion. HUVECs from different donors exhibit significant heterogeneity [47]. To reduce further variances in our experiments, we used a DMSO-differentiated PLB-985 cell line - devoid of the heterogeneity of primary cells, which is a well-known and suitable cellular model to study the functional responses of neutrophils [31, 48]. Furthermore, dPLB-985 could bind to E-selectin, whereas nondifferentiated PLB-985 could not, and this supports that it could be a suitable model for the assessment of adhesion. The pattern of dPLB-985 binding to HUVECs subjected to different pre-treatments was rather similar to that of the expression of E-selectin. rMASP-1 and thrombin enhanced adhesion, and elevated the E-selectin level only 6 hours after treatment - TNFalpha was superior in regard of both parameters, and at any times after induction. The possible role of E-selectin in this static model of dPLB-985 binding to HUVECs is further supported by the inhibition of adhesion by soluble E-selectin. To assess the adhesion force between rMASP-1-treated HUVECs and dPLB-985 cells, we utilized the recently introduced computer controlled micropipette method [34, 35]. Previously, it was applied to measure the adherence of 
macrophages and dendritic cells to fibrinogen, and to poly-L-Lys-graft-PEG. The adhesion force was in the same order of magnitude [35] as we found in the case of HUVECs/dPLB-985 cells. Our present data suggest that rMASP-1 induced high adhesion forces between dPLB-985 cells and HUVECs. The greatest difference - compared to untreated HUVECs - was measured at the highest detaching forces. This means that the number of firmly adherent cells increased significantly.

The complement system is a powerful defense mechanism for eliminating pathogens and necrotic debris. However, one of its main functions, the opsonization of unwanted particles, requires the presence of phagocytic cells. We found that complement MASP-1 can enhance the adhesion between neutrophils and endothelial cells in a direct fashion, and may thereby accelerate the recruitment of neutrophils at the scene of infection or tissue damage. This is in agreement also with our previous finding that MASP-1 increases the production of pro-inflammatory cytokines, such as of IL-6 and IL-8. Enhanced chemotaxis and adhesion may boost neutrophil functions; however, this hypothesis should be tested by further research.

\section{Conflict of interest}

BS is a founder of CellSorter, the startup company that developed the computer controlled micropipette. The other authors declare that they have no conflicts of interest with the contents of this article.

\section{Author contributions}

PKJ and ES contributed equally to this work. PKJ, ES, EK, MLD and LC harvested and cultured HUVECs and conducted most of the experiments. PKJ, ES and LC analyzed the results, and wrote the paper. JR organized and prepared the fresh umbilical cords obtained during normal delivery of healthy neonates for harvesting HUVECs. RUS and BS conducted and analyzed the experiments with the computer controlled micropipette. JD and PG prepared rMASP-1 and revised the manuscript. ZD conducted qPCR experiments. MG supervised Amplex Ultra Red based fluorescence method. All authors reviewed the results and approved the final version of the manuscript.

\section{Acknowledgments}

The authors thank Miklós Sándor Zoltán Kellermayer for revising the adhesion measurements at Department of Biophysics and Radiation Biology, Semmelweis University, Budapest, Hungary. This work was supported by Grants NK100834, NK100769, NK108642 and K100684 (OTKA, Hungarian Scientific Research Fund). PG, BS and LC were supported by MedinProt Synergy project. BS was supported by the Bolyai Scholarship of the Hungarian Academy of Sciences. 


\section{References}

[1] D. Heja, V. Harmat, K. Fodor, M. Wilmanns, J. Dobo, K.A. Kekesi, P. Zavodszky, P. Gal, G. Pal, Monospecific inhibitors show that both mannan-binding lectin-associated serine protease-1 (MASP-1) and -2 Are essential for lectin pathway activation and reveal structural plasticity of MASP-2, The Journal of biological chemistry, 287 (2012) 20290-20300.

[2] D. Heja, A. Kocsis, J. Dobo, K. Szilagyi, R. Szasz, P. Zavodszky, G. Pal, P. Gal, Revised mechanism of complement lectin-pathway activation revealing the role of serine protease MASP-1 as the exclusive activator of MASP-2, Proceedings of the National Academy of Sciences of the United States of America, 109 (2012) 10498-10503.

[3] M. Megyeri, V. Harmat, B. Major, A. Vegh, J. Balczer, D. Heja, K. Szilagyi, D. Datz, G. Pal, P. Zavodszky, P. Gal, J. Dobo, Quantitative characterization of the activation steps of mannan-binding lectin (MBL)-associated serine proteases (MASPs) points to the central role of MASP-1 in the initiation of the complement lectin pathway, The Journal of biological chemistry, 288 (2013) 8922-8934.

[4] T.R. Kjaer, S. Thiel, G.R. Andersen, Toward a structure-based comprehension of the lectin pathway of complement, Molecular immunology, 56 (2013) 222-231.

[5] M. Matsushita, Y. Endo, T. Fujita, Structural and functional overview of the lectin complement pathway: its molecular basis and physiological implication, Archivum immunologiae et therapiae experimentalis, 61 (2013) 273-283.

[6] J. Dobo, V. Schroeder, L. Jenny, L. Cervenak, P. Zavodszky, P. Gal, Multiple roles of complement MASP-1 at the interface of innate immune response and coagulation, Molecular immunology, 61 (2014) 6978.

[7] J. Dobo, B. Major, K.A. Kekesi, I. Szabo, M. Megyeri, K. Hajela, G. Juhasz, P. Zavodszky, P. Gal, Cleavage of kininogen and subsequent bradykinin release by the complement component: mannose-binding lectin-associated serine protease (MASP)-1, PloS one, 6 (2011) e20036.

[8] L. Jenny, J. Dobo, P. Gal, V. Schroeder, MASP-1 of the complement system promotes clotting via prothrombin activation, Molecular immunology, 65 (2015) 398-405.

[9] A. Krarup, K.C. Gulla, P. Gal, K. Hajela, R.B. Sim, The action of MBL-associated serine protease 1 (MASP1) on factor XIII and fibrinogen, Biochimica et biophysica acta, 1784 (2008) 1294-1300.

[10] K. Hess, R. Ajjan, F. Phoenix, J. Dobo, P. Gal, V. Schroeder, Effects of MASP-1 of the complement system on activation of coagulation factors and plasma clot formation, PloS one, 7 (2012) e35690.

[11] M. Megyeri, V. Mako, L. Beinrohr, Z. Doleschall, Z. Prohaszka, L. Cervenak, P. Zavodszky, P. Gal, Complement protease MASP-1 activates human endothelial cells: PAR4 activation is a link between complement and endothelial function, J Immunol, 183 (2009) 3409-3416.

[12] M. Megyeri, P.K. Jani, E. Kajdacsi, J. Dobo, E. Schwaner, B. Major, J. Rigo, Jr., P. Zavodszky, S. Thiel, L. Cervenak, P. Gal, Serum MASP-1 in complex with MBL activates endothelial cells, Molecular immunology, 59 (2014) 39-45.

[13] P.K. Jani, E. Kajdacsi, M. Megyeri, J. Dobo, Z. Doleschall, K. Futosi, C.I. Timar, A. Mocsai, V. Mako, P. Gal, L. Cervenak, MASP-1 induces a unique cytokine pattern in endothelial cells: a novel link between complement system and neutrophil granulocytes, PloS one, 9 (2014) e87104. 
[14] W.C. Aird, Phenotypic heterogeneity of the endothelium: I. Structure, function, and mechanisms, Circulation research, 100 (2007) 158-173.

[15] W.C. Aird, Phenotypic heterogeneity of the endothelium: II. Representative vascular beds, Circulation research, 100 (2007) 174-190.

[16] R. Hattori, K.K. Hamilton, R.D. Fugate, R.P. McEver, P.J. Sims, Stimulated secretion of endothelial von Willebrand factor is accompanied by rapid redistribution to the cell surface of the intracellular granule membrane protein GMP-140, The Journal of biological chemistry, 264 (1989) 7768-7771.

[17] M.G. Rondaij, R. Bierings, A. Kragt, J.A. van Mourik, J. Voorberg, Dynamics and plasticity of WeibelPalade bodies in endothelial cells, Arteriosclerosis, thrombosis, and vascular biology, 26 (2006) 1002-1007.

[18] O. Abbassi, T.K. Kishimoto, L.V. McIntire, D.C. Anderson, C.W. Smith, E-selectin supports neutrophil rolling in vitro under conditions of flow, The Journal of clinical investigation, 92 (1993) 2719-2730.

[19] G. Walz, A. Aruffo, W. Kolanus, M. Bevilacqua, B. Seed, Recognition by ELAM-1 of the sialyl-Lex determinant on myeloid and tumor cells, Science, 250 (1990) 1132-1135.

[20] M.G. Tonnesen, Neutrophil-endothelial cell interactions: mechanisms of neutrophil adherence to vascular endothelium, The Journal of investigative dermatology, 93 (1989) 53S-58S.

[21] M.L. Dustin, R. Rothlein, A.K. Bhan, C.A. Dinarello, T.A. Springer, Induction by IL 1 and interferongamma: tissue distribution, biochemistry, and function of a natural adherence molecule (ICAM-1). J. Immunol. 1986. 137: 245-254, J Immunol, 186 (2011) 5024-5033.

[22] J.S. Pober, L.A. Lapierre, A.H. Stolpen, T.A. Brock, T.A. Springer, W. Fiers, M.P. Bevilacqua, D.L. Mendrick, M.A. Gimbrone, Jr., Activation of cultured human endothelial cells by recombinant lymphotoxin: comparison with tumor necrosis factor and interleukin 1 species, J Immunol, 138 (1987) 3319-3324.

[23] T.M. Carlos, J.M. Harlan, Leukocyte-endothelial adhesion molecules, Blood, 84 (1994) 2068-2101.

[24] M.J. Elices, L. Osborn, Y. Takada, C. Crouse, S. Luhowskyj, M.E. Hemler, R.R. Lobb, VCAM-1 on activated endothelium interacts with the leukocyte integrin VLA-4 at a site distinct from the VLA4/fibronectin binding site, Cell, 60 (1990) 577-584.

[25] M. Shimaoka, C. Lu, R.T. Palframan, U.H. von Andrian, A. McCormack, J. Takagi, T.A. Springer, Reversibly locking a protein fold in an active conformation with a disulfide bond: integrin alphaL I domains with high affinity and antagonist activity in vivo, Proceedings of the National Academy of Sciences of the United States of America, 98 (2001) 6009-6014.

[26] P.W. Thompson, A.M. Randi, A.J. Ridley, Intercellular adhesion molecule (ICAM)-1, but not ICAM-2, activates RhoA and stimulates c-fos and rhoA transcription in endothelial cells, J Immunol, 169 (2002) 1007 1013.

[27] F. McLaughlin, B.P. Hayes, C.M. Horgan, J.E. Beesley, C.J. Campbell, A.M. Randi, Tumor necrosis factor (TNF)-alpha and interleukin (IL)-1beta down-regulate intercellular adhesion molecule (ICAM)-2 expression on the endothelium, Cell adhesion and communication, 6 (1998) 381-400.

[28] G. Ambrus, P. Gal, M. Kojima, K. Szilagyi, J. Balczer, J. Antal, L. Graf, A. Laich, B.E. Moffatt, W. Schwaeble, R.B. Sim, P. Zavodszky, Natural substrates and inhibitors of mannan-binding lectin-associated serine protease-1 and -2: a study on recombinant catalytic fragments, J Immunol, 170 (2003) 1374-1382.

[29] J. Dobo, V. Harmat, E. Sebestyen, L. Beinrohr, P. Zavodszky, P. Gal, Purification, crystallization and preliminary X-ray analysis of human mannose-binding lectin-associated serine protease-1 (MASP-1) 
catalytic region, Acta crystallographica. Section F, Structural biology and crystallization communications, 64 (2008) 781-784.

[30] M. Oroszlan, E. Herczenik, S. Rugonfalvi-Kiss, A. Roos, A.J. Nauta, M.R. Daha, I. Gombos, I. Karadi, L. Romics, Z. Prohaszka, G. Fust, L. Cervenak, Proinflammatory changes in human umbilical cord vein endothelial cells can be induced neither by native nor by modified CRP, International immunology, 18 (2006) 871-878.

[31] K.A. Tucker, M.B. Lilly, L. Heck, Jr., T.A. Rado, Characterization of a new human diploid myeloid leukemia cell line (PLB-985) with granulocytic and monocytic differentiating capacity, Blood, 70 (1987) 372-378.

[32] A. Donko, E. Ruisanchez, A. Orient, B. Enyedi, R. Kapui, Z. Peterfi, X. de Deken, Z. Benyo, M. Geiszt, Urothelial cells produce hydrogen peroxide through the activation of Duox1, Free radical biology \& medicine, 49 (2010) 2040-2048.

[33] V. Mako, J. Czucz, Z. Weiszhar, E. Herczenik, J. Matko, Z. Prohaszka, L. Cervenak, Proinflammatory activation pattern of human umbilical vein endothelial cells induced by IL-1beta, TNF-alpha, and LPS, Cytometry. Part A : the journal of the International Society for Analytical Cytology, 77 (2010) 962-970.

[34] Z. Kornyei, S. Beke, T. Mihalffy, M. Jelitai, K.J. Kovacs, Z. Szabo, B. Szabo, Cell sorting in a Petri dish controlled by computer vision, Scientific reports, 3 (2013) 1088.

[35] R. Salanki, C. Hos, N. Orgovan, B. Peter, N. Sandor, Z. Bajtay, A. Erdei, R. Horvath, B. Szabo, Single cell adhesion assay using computer controlled micropipette, PloS one, 9 (2014) e111450.

[36] T.R. Kjaer, T.M. Le le, J.S. Pedersen, B. Sander, M.M. Golas, J.C. Jensenius, G.R. Andersen, S. Thiel, Structural insights into the initiating complex of the lectin pathway of complement activation, Structure, 23 (2015) 342-351.

[37] J.H. Cleator, W.Q. Zhu, D.E. Vaughan, H.E. Hamm, Differential regulation of endothelial exocytosis of P-selectin and von Willebrand factor by protease-activated receptors and cAMP, Blood, 107 (2006) 27362744.

[38] P. Kubes, S. Kanwar, Histamine induces leukocyte rolling in post-capillary venules. A P-selectinmediated event, J Immunol, 152 (1994) 3570-3577.

[39] P.S. Frenette, R.C. Johnson, R.O. Hynes, D.D. Wagner, Platelets roll on stimulated endothelium in vivo: an interaction mediated by endothelial P-selectin, Proceedings of the National Academy of Sciences of the United States of America, 92 (1995) 7450-7454.

[40] A.M. Olofsson, K.E. Arfors, L. Ramezani, B.A. Wolitzky, E.C. Butcher, U.H. von Andrian, E-selectin mediates leukocyte rolling in interleukin-1-treated rabbit mesentery venules, Blood, 84 (1994) 2749-2758.

[41] M. Pudelko, J. Bull, H. Kunz, Chemical and chemoenzymatic synthesis of glycopeptide selectin ligands containing sialyl Lewis X structures, Chembiochem : a European journal of chemical biology, 11 (2010) 904-930.

[42] G.S. Kansas, Selectins and their ligands: current concepts and controversies, Blood, 88 (1996) 32593287.

[43] E.B. Lomakina, R.E. Waugh, Adhesion between human neutrophils and immobilized endothelial ligand vascular cell adhesion molecule 1: divalent ion effects, Biophysical journal, 96 (2009) 276-284. 
[44] A. Pietersma, B.C. Tilly, M. Gaestel, N. de Jong, J.C. Lee, J.F. Koster, W. Sluiter, p38 mitogen activated protein kinase regulates endothelial VCAM-1 expression at the post-transcriptional level, Biochemical and biophysical research communications, 230 (1997) 44-48.

[45] C. Volanti, G. Gloire, A. Vanderplasschen, N. Jacobs, Y. Habraken, J. Piette, Downregulation of ICAM-1 and VCAM-1 expression in endothelial cells treated by photodynamic therapy, Oncogene, 23 (2004) 8649-8658.

[46] U.J. Soh, M.R. Dores, B. Chen, J. Trejo, Signal transduction by protease-activated receptors, British journal of pharmacology, 160 (2010) 191-203.

[47] P. Kiszel, V. Mako, Z. Prohaszka, L. Cervenak, Interleukin-6 -174 promoter polymorphism does not influence IL-6 production after LPS and IL-1 beta stimulation in human umbilical cord vein endothelial cells, Cytokine, 40 (2007) 17-22.

[48] G.L. Petheo, A. Orient, M. Barath, I. Kovacs, B. Rethi, A. Lanyi, A. Rajki, E. Rajnavolgyi, M. Geiszt, Molecular and functional characterization of Hv1 proton channel in human granulocytes, PloS one, 5 (2010) e14081.

\section{Table 1}

Primers for the analysis of mRNAs of the adhesion molecules.

\begin{tabular}{|c|c|c|}
\hline \multirow{2}{*}{$\begin{array}{c}\boldsymbol{E}- \\
\text { selectin }\end{array}$} & forward: & 5'-tcaagtgtgagcaaattgtgaac-3' \\
\cline { 2 - 3 } & reverse: & 5'-attctccagaggacatacactgc-3' \\
\hline \multirow{2}{*}{ ICAM-1 } & forward: & 5'-acagtcacctatggcaacgac-3' \\
\cline { 2 - 3 } & reverse: & 5'-gtcactgtctgcagtgtctcct-3' \\
\hline \multirow{2}{*}{ ICAM-2 } & forward: & 5'-acagccacattcaacagcac-3' \\
\cline { 2 - 3 } & reverse: & 5'-agatgtcacgaacagggacag-3' \\
\hline \multirow{2}{*}{ VCAM-1 } & forward: & 5'-tgaccttcatcctaccattga-3' \\
\cline { 2 - 3 } & reverse: & 5'-gcatgtcatattcacagaactgc-3' \\
\hline
\end{tabular}


Fig. 1.

A

\section{B}
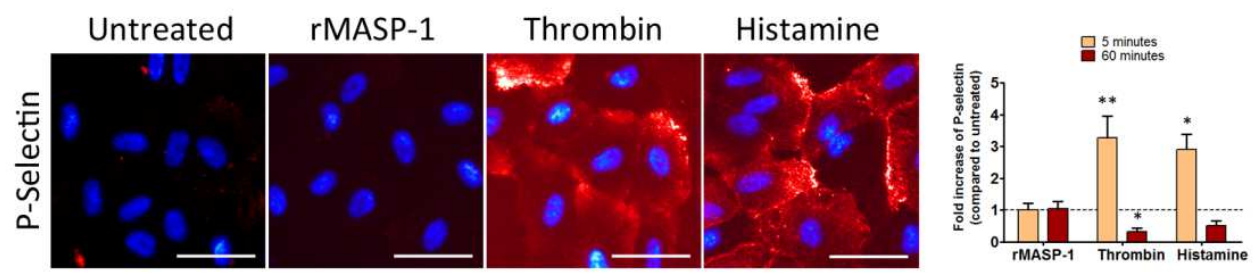

rMASP-1
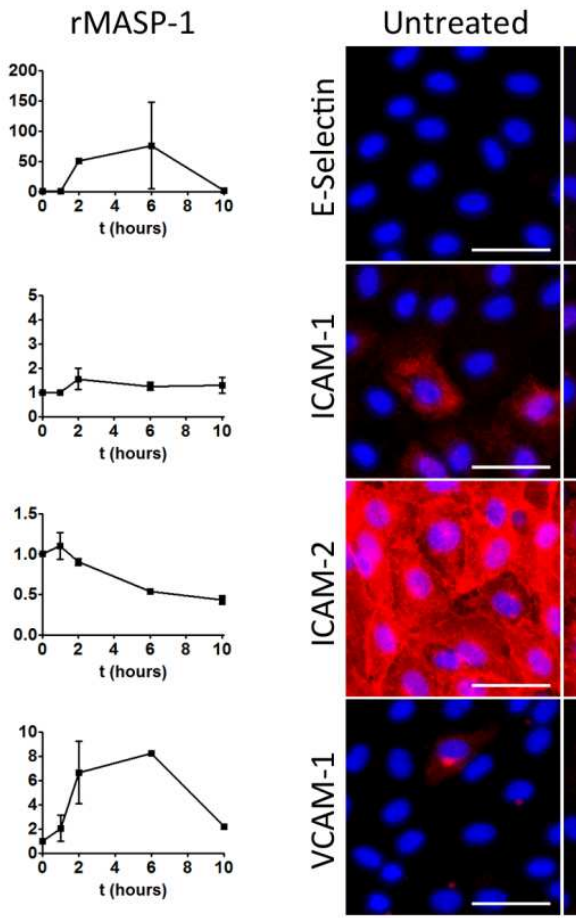

rMASP-1

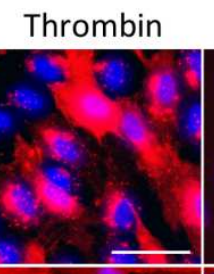

TNFalpha
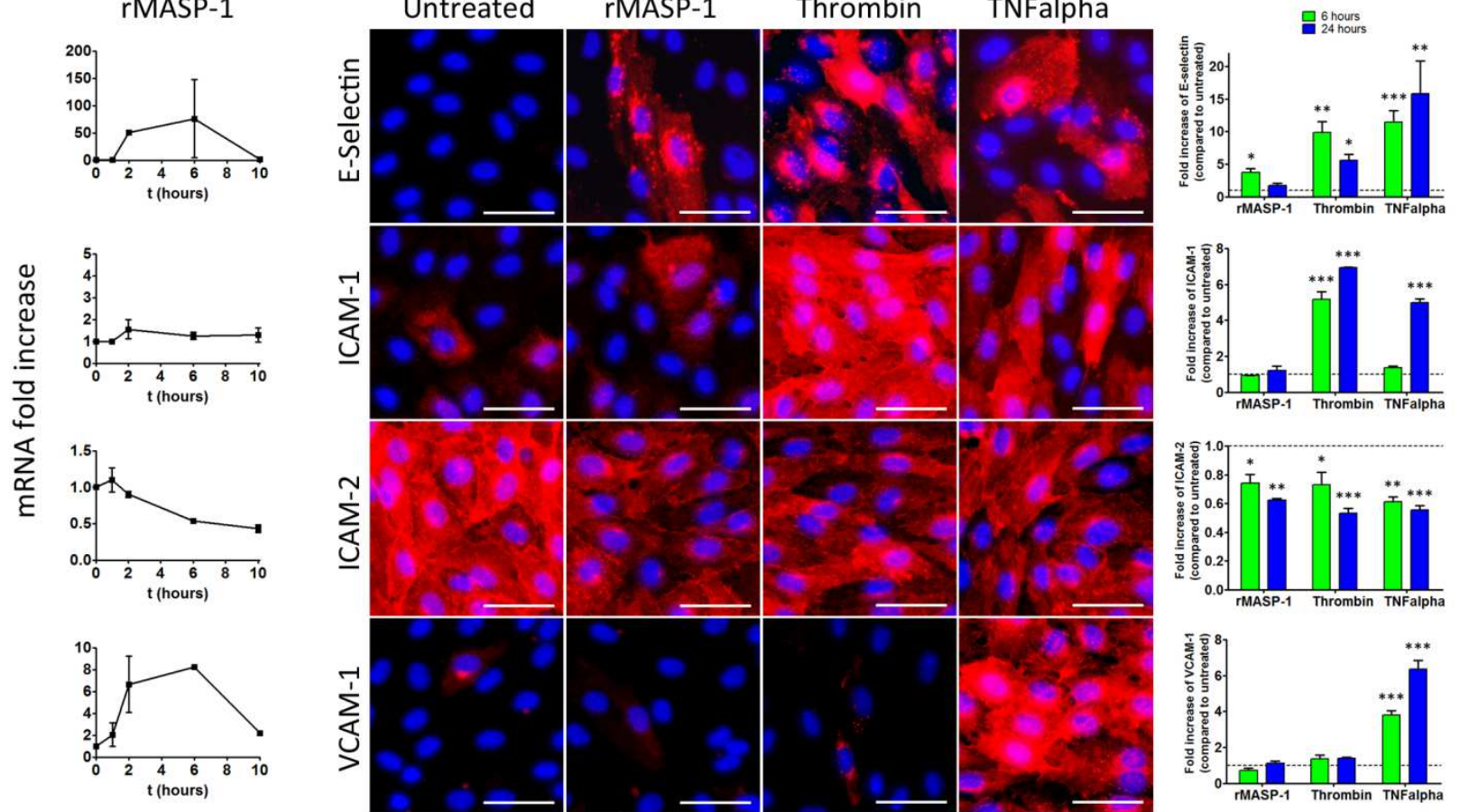

Fig. 1. Adhesion molecule patterns induced by rMASP-1 on HUVECs. (A) HUVECs were treated with 2 $\mu \mathrm{M}$ MASP-1 for 1, 2, 6, or 10 hours. Then, the cells were lysed and stored in TRI-reagent, total mRNA was purified, and qPCR performed. Values of the quantities of the adhesion molecule mRNAs in three independent experiments were normalized for that of GAPDH, used as internal control. P-selectin was not analyzed by qPCR, because of its instant secretion from pre-formed granules. (B) HUVECs were treated with $2 \mu \mathrm{M}$ MASP-1, $300 \mathrm{nM}$ thrombin, $50 \mu \mathrm{M}$ histamine, or $10 \mathrm{ng} / \mathrm{mL} \mathrm{TNF} \alpha$ for 5 minutes (P-selectin), or for 6 hours (in the case of all other adhesion molecules). Then, cells were fixed and stained with mouse monoclonal anti-adhesion molecule antibodies, followed by goat-anti-mouse Alexa 568 (red) and Hoechst 33342 (blue) as nuclear staining. Each subfigure of panel B is a representative photo of three independent experiments. (C) HUVECs were treated in 96-well plates as described in panel (B), and cellular ELISA was performed. The values represent the ratio of the optical density of treated vs. non-treated wells, and the difference was analyzed by ANOVA with Tukey's post-test. Dotted lines represent the untreated control levels. The values were calculated as the mean (+/-SEM) of three independent experiments. *: $\mathrm{p}<0.05$, **: $\mathrm{p}<0.01, * * *: \mathrm{p}<0.001$. 
Fig. 2.
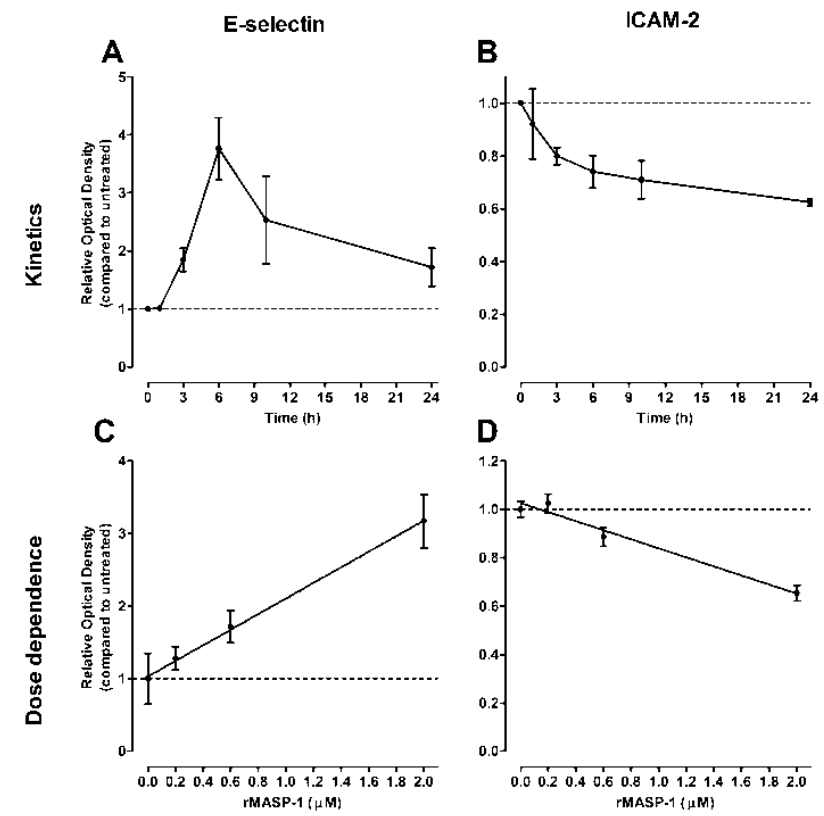

Fig. 2. Dose- and time- dependence of E-selectin and ICAM-2 expression induced by rMASP-1. HUVECs were treated with $2 \mu \mathrm{M}$ of rMASP-1 for $1,3,6,10$, and 24 hours (A and B), or with $0,0,2,0,6$, $2 \mu \mathrm{M}$ MASP-1 for 6 hours (C) and 24 hours (D). Then, relative expression (compared to untreated controls of E-selectin and ICAM-2) was measured by cell-based ELISA. Dotted lines represent the untreated control levels. The values were calculated as the mean (+/-SEM) of three independent experiments. One-way ANOVA has been performed for each subfigure. In the case of kinetic measurements $\mathrm{p}<0.01$ for $\mathrm{A}$ and $\mathrm{p}<0.05$ for $\mathrm{B}$, and of dose-dependence experiments, ANOVA ( $<<0.05$ for $\mathrm{C}$ and $\mathrm{p}<0.001$ for $\mathrm{D}$ ) was supplemented with post-test for linear trend ( $r=0.55, \mathrm{p}<0.01$ for $\mathrm{C}$ and $\mathrm{r}=-0.69, \mathrm{p}<0.001$ for $\mathrm{D})$. 
Fig. 3.

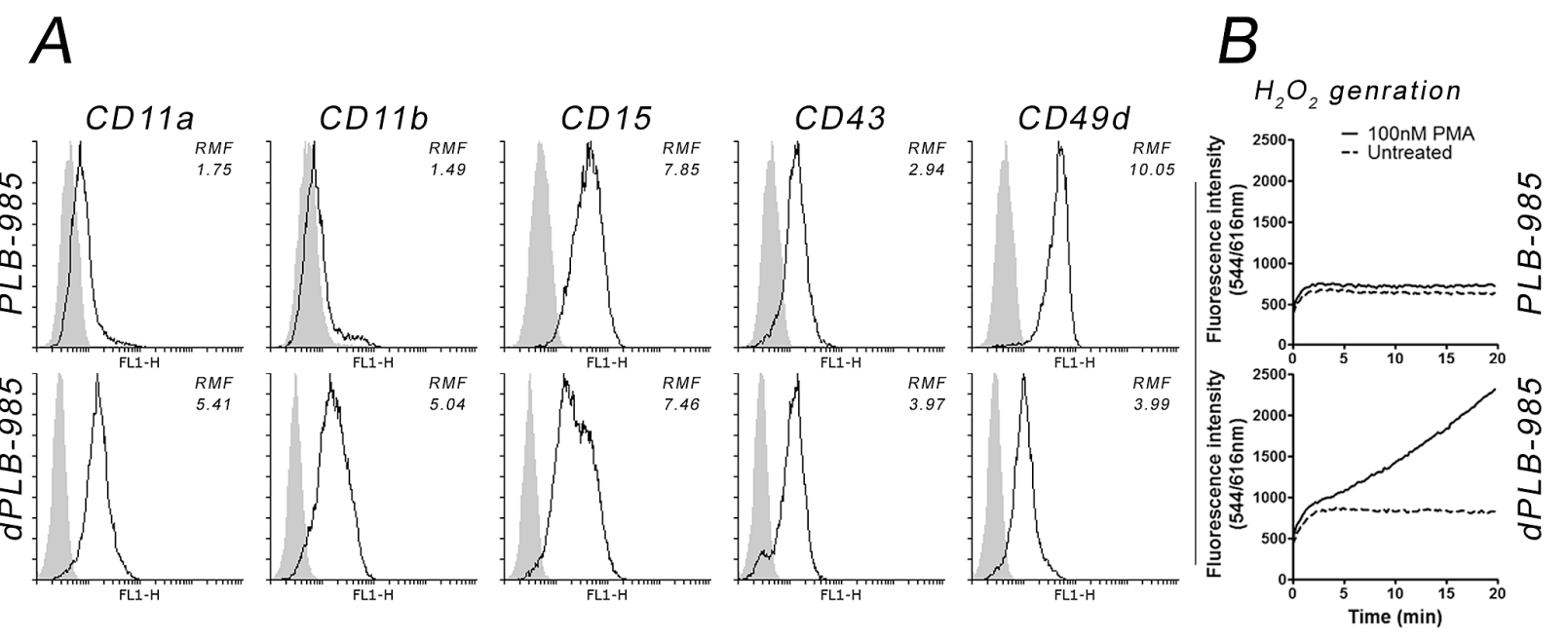

Fig. 3. The effects of PLB-985 cell differentiation on adhesion molecule pattern and hydrogen peroxyde generation. (A) PLB-985 cells or DMSO-differentiated PLB-985 cells were suspended in HBSS. Specific mouse antibodies or isotype controls were added to the cell suspensions and incubated for $30 \mathrm{~min}$, at $4{ }^{\circ} \mathrm{C}$. After washing, samples were fixed in $2 \%$ formaldehyde for 15 minutes, and suspended in PBS for flow cytometry analysis. $10^{4}$ cells were measured in each sample. RMF: Ratio of geometric mean fluorescence intensity values of labeled cells and of respective isotype controls. (B) HRP and Amplex Ultra Red ${ }^{\mathrm{TM}}$ fluorescence substrate was added to PLB-985 or dPLB-985 cells, which were then activated with $100 \mathrm{nM}$ PMA or left untreated. Fluorescence was recorded every 10 seconds for 20 minutes, and expressed as arbitrary units. One representative experiment out of three is shown. 
Fig. 4.
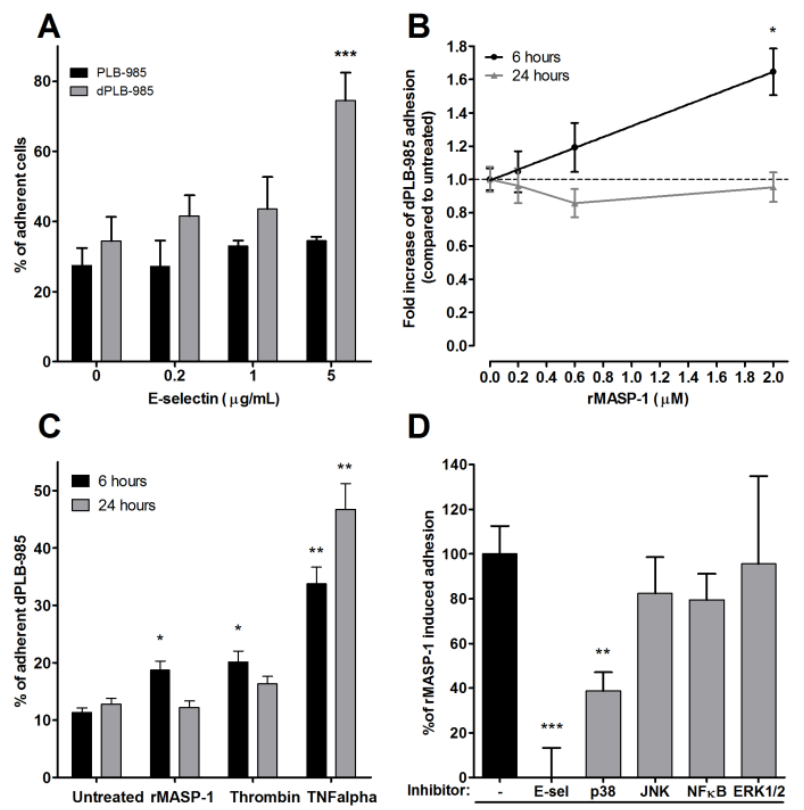

D

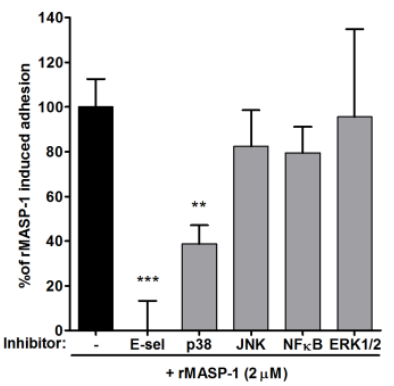

Fig. 4. The adhesion of dPLB-985 cells to E-selectin coated plates or to MASP-1-treated endothelial cells. (A) After 96-well plates had been pre-coated with $0,0.2,1$, or $5 \mu \mathrm{g} / \mathrm{mL}$ E-selectin, OG-488-labeled PLB-985 or dPLB-985 cells were added to the wells and incubated for 1 hour, at $37{ }^{\circ} \mathrm{C}$, in $5 \% \mathrm{CO}_{2}$ atmosphere. Then, the fluorescence of bound cells was detected after washing with HBSS. The number of bound cells in three independent measurements was calculated. (B, C) HUVECs were treated with 0, 0.2, 0.6 , and $2 \mu \mathrm{M}$ MASP-1 for 6 and 24 hours (B), with $2 \mu \mathrm{M}$ of rMASP-1, $300 \mathrm{nM}$ thrombin, or $10 \mathrm{ng} / \mathrm{mL}$ TNFalpha - or left untreated - for 6 , and 24 hours (C), and adhesion was measured as in panel A. (D) Adhesion of OG-488-labeled dPLB-985 to HUVECs treated with $2 \mu \mathrm{M}$ MASP-1 for 6 hours was inhibited either by pre-incubation of HUVECs with p38-MAPK, JNK, NFkB, or ERK 1/2 inhibitor for 30 minutes or by pre-incubation of dPLB-985 with soluble recombinant E-selectin for 30 minutes. Then adhesion was assessed as described above in panel A, and the inhibition is shown as the percentage of the MASP-1 induced adhesion. The values were calculated as the mean (+/-SEM) of at least three independent experiments. Two-way ANOVA was used for panel A, whereas one-way ANOVA has been performed for panel B, C and D. Dotted lines represent the untreated control levels. *: $p<0.05$, **: $p<0.01$, ***: $p<0.001$. 
Fig. 5.

A

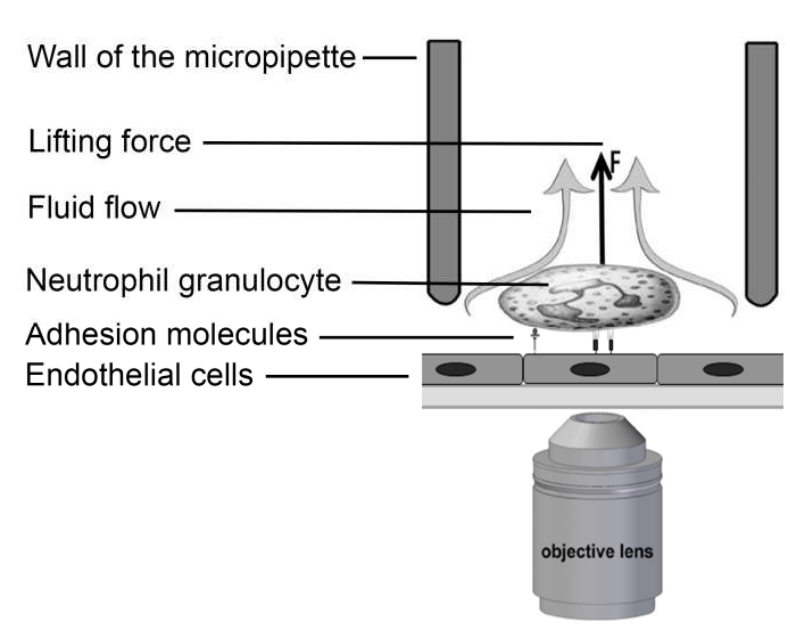

B

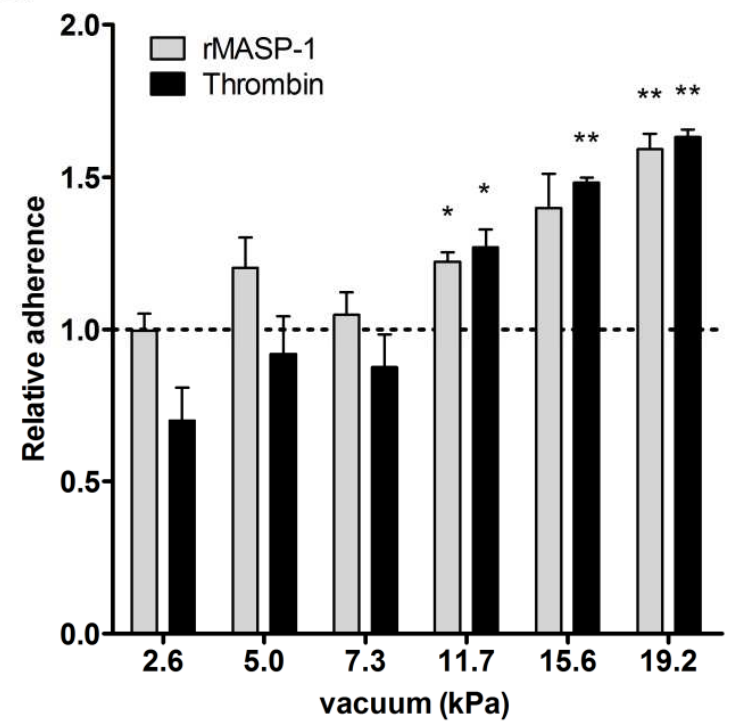

Fig. 5. Adhesion force measurement between cells using an automated micropipette. (A) Schematic representation of the hydrodynamic adhesion force measurement. OG-488 labeled dPLB-985 cells attached to the confluent layer of HUVECs were scanned and recognized by software in the microscopic images captured on a motorized inverted microscope. A glass micropipette was automatically positioned to each detected cell one by one. Cell adhesion was probed by the application of a precisely controlled fluid flow through the micropipette. (B) Adhesion force between dPLB-985 cells and MASP-1-treated endothelial cells. HUVECs were treated in Petri dishes with $2 \mu \mathrm{M}$ of rMASP-1, or $300 \mathrm{nM}$ thrombin for 6 hours, or with $10 \mathrm{ng} / \mathrm{mL}$ TNFalpha for 24 hours, or left untreated. Then, OG-488-labeled dPLB-985 cells were seeded onto the HUVECs (subjected to different pre-treatments) for 1 hour. The adhesion force between dPLB-985 cells and HUVECs was tested by the computer controlled micropipette method. The vacuum applied (expressed in $\mathrm{kPa}$ ) was proportional to the hydrodynamic lifting force acting on the targeted single cells. Significant deviation from the untreated values in the high-vacuum range of the plot means that the ratio of cells exhibiting firm adhesion was increased by treatment. The values were calculated as the ratio of bound cell number of treated and non treated samples at each vacuum pressure points, and shown as the mean (+/-SEM) of at least three independent experiments. One-way ANOVA has been performed for panel B. Dotted lines represent the untreated control levels. ${ }^{*}: \mathrm{p}<0.05,{ }^{* *}: \mathrm{p}<0.01$. 